\title{
Multiple finite Riemann zeta functions
}

\author{
by
}

\section{Kazufumi Kimoto (Okinawa), Nobushige Kurokawa (Tokyo), Sho Matsumoto (Fukuoka) and Masato Wakayama (Fukuoka)}

1. Introduction. The divisor function $\sigma_{k}(N):=\sum_{d \mid N} d^{k}$ is a basic multiplicative function and plays an important role from the beginning of the modern arithmetic study. In particular, $\sigma_{k}(N)$ appears in the Fourier coefficients of the (holomorphic) Eisenstein series $E_{k+1}(\tau)$. It is, however, not so common to regard $\sigma_{k}(N)$ as a sort of zeta function. In the present paper, we treat $\sigma_{k}(N)$ as a function of a complex variable $k=-s$. It is clear that when $N=0$ (or $N \rightarrow \infty$ ) we have $\sigma_{-s}(N) \rightarrow \zeta(s)$, the Riemann zeta function. There are at least two interpretations of $Z_{N}^{1}(s):=\sum_{n \mid N} n^{-s}=$ $\sigma_{-s}(N)$ as a zeta function in number theory:

- Fourier coefficients of real analytic Eisenstein series,

- Igusa zeta functions.

Concerning the first, we refer to Bump et al. [BCKV], where the socalled "local Riemann hypothesis" is studied. In the case of the real analytic Eisenstein series $E(s, \tau)$ for the modular group $\mathrm{SL}(2, \mathbb{Z})$, the $N$ th Fourier coefficient is essentially given by

$$
c_{N}(s, \tau):=Z_{N}^{1}(2 s-1) K_{s-1 / 2}(2 \pi N \operatorname{Im} \tau) e^{2 \pi i \operatorname{Re} \tau} .
$$

Hence it satisfies the local Riemann hypothesis: if $c_{N}(s, \tau)=0$ then $\operatorname{Re} s=$ $1 / 2$. For the second, the interpretation comes from the (global) Igusa zeta function $\zeta^{\text {Igusa }}(s, R)$ of a ring $R$ defined as

$$
\zeta^{\text {Igusa }}(s, R):=\sum_{m=1}^{\infty} \# \operatorname{Hom}_{\text {ring }}(R, \mathbb{Z} /(m)) m^{-s} .
$$

Then, in fact, it is easy to see that $Z_{N}^{1}(s)=\zeta^{\operatorname{Igusa}}(s, \mathbb{Z} /(N))$.

2000 Mathematics Subject Classification: Primary 11M36.

Key words and phrases: Riemann zeta function, divisor functions, Euler product, $q$-binomial coefficients, complete symmetric polynomial, powerful numbers. 
The purpose of the present paper is, first, to study the function defined by the series

$$
Z_{N}^{m}(s):=\sum_{n_{1}\left|n_{2}\right| \cdots\left|n_{m}\right| N}\left(n_{1} n_{2} \cdots n_{m}\right)^{-s} .
$$

We call $Z_{N}^{m}(s)$ the multiple finite Riemann zeta function of type $N$. We then study several basic properties of $Z_{N}^{m}(s)$ such as an Euler product, a functional equation, and an analogue of the Riemann hypothesis in an elementary way with the help of some $q$-series identity. We also study the limit case $Z_{\infty}^{m}(s):=\sum_{n_{1}|\cdots| n_{m}}\left(n_{1} \cdots n_{m}\right)^{-s}$.

Moreover, we generalize this zeta function in two directions. The first one is to increase the number of variables. We prove that the Euler product of a multi-variable version of $Z_{N}^{m}(s)$ can be expressed in terms of the complete symmetric polynomials with a remarkable specialization of variables (Theorem 3.2). The second is to add parameters indexed by a set of positive integers. For general parameters, it seems difficult to calculate an Euler product explicitly using symmetric functions with some meaningful specialization of variables. If we restrict ourselves to the one variable case, however, under a special but non-trivial specialization of parameters, we show that the corresponding multiple zeta functions can be written as a product of $\zeta(c s)$ with several constants $c$ determined by the given parameters and the Dirichlet series associated with generalized powerful numbers (see Section 4). Moreover, we determine the condition for the Dirichlet series associated with such generalized powerful numbers to be extendable as a meromorphic function to the entire plane $\mathbb{C}$ (see Theorem 4.8 and its corollary). As a consequence, most of such Dirichlet series are shown to have the imaginary axis as a natural boundary. The result is a generalization of the one in [IS]. In the last section, we give two remarks on the number of isomorphism classes of abelian groups and an analogous notion of Eisenstein series.

Throughout the paper, we denote the sets of all integers, positive integers, non-negative integers, real numbers, and complex numbers by $\mathbb{Z}, \mathbb{Z}_{>0}$, $\mathbb{Z}_{\geq 0}, \mathbb{R}$, and $\mathbb{C}$, respectively.

2. Multiple finite Riemann zeta function. We prove the fundamental properties of the multiple finite Riemann zeta function $Z_{N}^{m}(s)$ defined by (1.1) and discuss some related Dirichlet series. First we have

TheOREM 2.1. Let $N$ be a positive integer.

(i) (Euler product)

$$
Z_{N}^{m}(s)=\prod_{p: \text { prime }} \prod_{k=1}^{m} \frac{1-p^{-s\left(\operatorname{ord}_{p} N+k\right)}}{1-p^{-s k}},
$$


where $\operatorname{ord}_{p} N$ denotes the order of the p-factor in the prime decomposition of $N$.

(ii) (Functional equation) $Z_{N}^{m}(-s)=N^{m s} Z_{N}^{m}(s)$.

(iii) (Analogue of the Riemann hypothesis) All zeros of $Z_{N}^{m}(s)$ lie on the imaginary axis $\operatorname{Re} s=0$. More precisely, the zeros of $Z_{N}^{m}(s)$ are of the form

$$
s=\frac{2 \pi i n}{\left(\operatorname{ord}_{p} N+k\right) \log p} \quad \text { for } k=1, \ldots, m, p \mid N \text { and } n \in \mathbb{Z} \backslash\{0\} .
$$

Consequently, the order $\mathrm{Mult}^{m}(n, p, k)$ of the zero at the above value of $s$ is given by

$$
\begin{aligned}
& \operatorname{Mult}^{m}(n, p, k)= \\
& \quad \#\left\{(l, j): 1 \leq l \leq m, j \in \mathbb{Z} \backslash\{0\},\left(\operatorname{ord}_{p} N+k\right) j=\left(\operatorname{ord}_{p} N+l\right) n\right\} .
\end{aligned}
$$

(iv) (Special value) When $n$ is a positive integer one has $Z_{N}^{m}(-n) \in \mathbb{Z}$.

For the proof of the theorem the following lemma is crucial.

Lemma 2.2. Let $m$ be a positive integer. Then:

(i) For any integers $l \geq 0$,

$$
\sum_{d=0}^{l}\left[\begin{array}{c}
m-1+d \\
m-1
\end{array}\right]_{q} q^{d}=\left[\begin{array}{c}
m+l \\
m
\end{array}\right]_{q}
$$

where $\left[\begin{array}{l}n \\ k\end{array}\right]_{q}$ is the q-binomial coefficient defined by

$$
\left[\begin{array}{l}
n \\
k
\end{array}\right]_{q}=\prod_{j=1}^{k}\left(1-q^{n+1-j}\right) /\left(1-q^{j}\right) .
$$

(ii) For $|x|<1,|q|<1$,

$$
\sum_{d=0}^{\infty}\left[\begin{array}{c}
m+d \\
m
\end{array}\right]_{q} x^{d}=\prod_{k=0}^{m} \frac{1}{1-q^{k} x} .
$$

Proof. We prove (2.2) by induction on $l$. When $l=0,(2.2)$ clearly holds. Suppose that it holds for $l$. Then we see that

$$
\sum_{d=0}^{l+1}\left[\begin{array}{c}
m-1+d \\
m-1
\end{array}\right]_{q} q^{d}=\left[\begin{array}{c}
m+l \\
m
\end{array}\right]_{q}+\left[\begin{array}{c}
m+l \\
m-1
\end{array}\right]_{q} q^{l+1}=\left[\begin{array}{c}
m+l+1 \\
m
\end{array}\right]_{q},
$$

whence $(2.2)$ is also true for $l+1$.

The second formula (2.3) can be proved in the same manner by induction, but on $m$. (It is also obtained from the so-called $q$-binomial theorem, see, e.g., $[\mathrm{AAR}]$. 
Proof of Theorem 2.1. The functional equation is easily seen from the definition. Actually, we have

$$
\begin{aligned}
Z_{N}^{m}(-s) & =\sum_{n_{1}\left|n_{2}\right| \cdots\left|n_{m}\right| N}\left(n_{1} n_{2} \cdots n_{m}\right)^{s}=N^{m s} \sum_{n_{1}\left|n_{2}\right| \cdots\left|n_{m}\right| N}\left(\frac{n_{1}}{N} \frac{n_{2}}{N} \cdots \frac{n_{m}}{N}\right)^{s} \\
& =N^{m s} Z_{N}^{m}(s) .
\end{aligned}
$$

To prove (2.1), we show that $Z_{N}^{m}(s)$ is multiplicative with respect to $N$. Suppose $N$ and $M$ are co-prime. Then

$$
\begin{aligned}
Z_{N M}^{m}(s) & =\sum_{n_{1}|\cdots| n_{m} \mid N M}\left(n_{1} \cdots n_{m}\right)^{-s} \\
& =\sum_{c_{1}|\cdots| c_{m} \mid N}\left(c_{1} \cdots c_{m}\right)^{-s} \sum_{d_{1}|\cdots| d_{m} \mid M}\left(d_{1} \cdots d_{m}\right)^{-s}=Z_{N}^{m}(s) Z_{M}^{m}(s),
\end{aligned}
$$

because every divisor $n$ of $N M$ is uniquely written as $n=c d$, where $c \mid N$ and $d \mid M$. Hence, to get the Euler product expression $(2.1)$ of $Z_{N}^{m}(s)$ it suffices to consider the case where $N$ is a power of prime $p$. In this case, one proves that

$$
Z_{p^{l}}^{m}(s)=\prod_{k=1}^{m} \frac{1-p^{-(l+k) s}}{1-p^{-s k}}
$$

by induction on $m$ as follows: It is clear for $m=1$. Suppose (2.4) is true for $m-1$. Then

$$
\begin{aligned}
Z_{p^{l}}^{m}(s) & =\sum_{0 \leq j_{1} \leq \cdots \leq j_{m} \leq l} p^{-\left(j_{1}+\cdots+j_{m}\right) s}=\sum_{j_{m}=0}^{l} Z_{p^{j_{m}}}^{m-1}(s) p^{-j_{m} s} \\
& =\sum_{d=0}^{l} \prod_{k=1}^{m-1} \frac{1-p^{-(d+k) s}}{1-p^{-s k}} p^{-d s}=\sum_{d=0}^{l}\left[\begin{array}{c}
m-1+d \\
d
\end{array}\right]_{p^{-s}} p^{-d s} .
\end{aligned}
$$

Therefore the assertion follows immediately from (2.2). This proves (2.4), whence the Euler product for $Z_{N}^{m}(s)$ follows.

Using (2.1), we observe that the meromorphic function $Z_{N}^{m}(s)$ may have zeros at each $s=2 \pi i n /\left(\operatorname{ord}_{p} N+k\right) \log p$ for $k=1, \ldots, m, p \mid N$ and $n \in \mathbb{Z}$. Note, however, that since

$$
Z_{N}^{m}(0)=\prod_{p: \text { prime }}\left(\begin{array}{c}
\operatorname{ord}_{p} N+m \\
m
\end{array}\right) \neq 0,
$$

$s=0$ is not a zero of $Z_{N}^{m}(s)$. Suppose now that

$$
\frac{2 \pi i n}{\left(\operatorname{ord}_{p} N+k\right) \log p}=\frac{2 \pi i m}{\left(\operatorname{ord}_{q} N+l\right) \log q}
$$

for some $1 \leq k, l \leq m, p, q \mid N$ and $n, j \in \mathbb{Z} \backslash\{0\}$. Then $p^{\left(\operatorname{ord}_{p} N+k\right) j}=$ $q^{\left(\operatorname{ord}_{q} N+l\right) n}$. This immediately shows that $p=q$ and $\left(\operatorname{ord}_{p} N+k\right) j=$ 
$\left(\operatorname{ord}_{p} N+l\right) n$. Hence the order of zero at $s=2 \pi i n /\left(\operatorname{ord}_{p} N+k\right) \log p$ is given by $\operatorname{Mult}^{m}(n, p, k)$. Obviously, $\operatorname{Mult}^{1}(n, p, 1)=1$.

The last claim about the special value of $Z_{N}^{m}(s)$ is clear from the definition.

REMARK 2.1. Note that $Z_{p^{l}}^{m}(s)=Z_{p^{m}}^{l}(s)$ from (2.4).

We next consider a Dirichlet series defined via the multiple finite Riemann zeta functions. Put

$$
\zeta^{m}(s, t):=\sum_{n=1}^{\infty} Z_{n}^{m}(s) n^{-t} .
$$

Then, from $(2.3), \zeta^{m}(s, t)$ has the Euler product

$$
\zeta^{m}(s, t)=\prod_{p: \text { prime }}\left(\sum_{l=0}^{\infty} Z_{p^{l}}^{m}(s) p^{-l t}\right)=\prod_{k=0}^{m} \zeta(s k+t) .
$$

We give a few examples:

EXAMPLE 2.1. It is well known that

$$
\zeta^{1}(-k, t)=\sum_{n=1}^{\infty} \sigma_{k}(n) n^{-t}=\zeta(t) \zeta(t-k) \quad \text { for } \operatorname{Re} t>k+1 .
$$

Also, by $(2.5), \zeta^{m}(0, t)=\zeta(t)^{m+1}$ for $\operatorname{Re} t>1$.

ExAmple 2.2. Let $l$ be a positive integer. For $\operatorname{Re} t>1-l$, we have

$$
\zeta^{m}(l, t+l)=\prod_{k=1}^{m+1} \zeta(t+l k)
$$

As $m \rightarrow \infty$, we have

$$
\lim _{m \rightarrow \infty} \zeta^{m}(l, t+l)=\prod_{k=1}^{\infty} \zeta(t+l k),
$$

which is the higher Riemann zeta function $\zeta_{l \infty}(s)$ studied in $[\mathrm{KMW}]$ (see also $[\mathrm{KW}])$. Note that $\zeta_{l \infty}(s)$ satisfies a functional equation.

3. Multivariable version. We study a generalization of $Z_{N}^{m}(s)$. For $\gamma=\left(\gamma_{1}, \ldots, \gamma_{m}\right) \in \mathbb{Z}_{>0}^{m}$ and $N \in \mathbb{Z}_{>0}$, define

$$
Z_{N}^{\gamma}\left(t_{1}, \ldots, t_{m}\right):=\sum_{n_{m}^{\gamma_{m}}|\cdots| n_{1}^{\gamma_{1}} \mid N} n_{1}^{-\gamma_{1} t_{1}} \cdots n_{m}^{-\gamma_{m} t_{m}} .
$$

This is multiplicative with respect to $N$. Notice that $Z_{N}^{m}(s)=Z_{N}^{\gamma}(s, \ldots, s)$ when $\gamma=\left(1^{m}\right)=(\overbrace{1, \ldots, 1}^{m})$. We can prove the following lemma in a similar way to Theorem 2.1 . 
Lemma 3.1. For $l \geq 0$, define

$$
G_{l}^{\gamma}\left(q_{1}, \ldots, q_{m}\right)=\sum_{\substack{l \geq \lambda_{1} \geq \cdots \geq \lambda_{m} \geq 0 \\ \gamma_{j} \mid \lambda_{j}}} q_{1}^{\lambda_{1}} \cdots q_{m}^{\lambda_{m}},
$$

where the sum is taken over all partitions $\lambda=\left(\lambda_{1}, \lambda_{2}, \ldots\right)$ of length $\leq m$ such that $\lambda_{1} \leq l$ and $\gamma_{j} \mid \lambda_{j}$ for $1 \leq j \leq m$. Then the Euler product of $Z_{N}^{\gamma}\left(t_{1}, \ldots, t_{m}\right)$ is given as

$$
Z_{N}^{\gamma}\left(t_{1}, \ldots, t_{m}\right)=\prod_{p: \text { prime }} G_{\operatorname{ord}_{p} N}^{\gamma}\left(p^{-t_{1}}, \ldots, p^{-t_{m}}\right) .
$$

REMARK 3.1. Let $f\left(n_{1}, \ldots, n_{m}\right)$ be a multiplicative function with respect to each $n_{j}$. We define a multiple zeta function by

$$
Z_{N}^{\gamma}(f)=\sum_{n_{m}^{\gamma_{m}}|\cdots| n_{1}^{\gamma_{1}} \mid N} f\left(n_{1}, \ldots, n_{m}\right) .
$$

Then one can show that $Z_{N}^{\gamma}(f)$ is multiplicative, whence has the Euler product $Z_{N}^{\gamma}(f)=\prod_{p: \text { prime }} Z_{p^{\text {ord } p N}}^{\gamma}(f)$.

We first look at the simplest case $\gamma=\left(1^{m}\right)$. We abbreviate respectively $Z_{N}^{\left(1^{m}\right)}\left(t_{1}, \ldots, t_{m}\right), G_{l}^{\left(1^{m}\right)}\left(q_{1}, \ldots, q_{m}\right)$ to $Z_{N}^{m}\left(t_{1}, \ldots, t_{m}\right), G_{l}^{m}\left(q_{1}, \ldots, q_{m}\right)$.

THEOREM 3.2. Let

$$
h_{j}\left(x_{1}, \ldots, x_{m}\right)=\sum_{\substack{i_{1}+\cdots+i_{m}=j \\ i_{k} \in \mathbb{Z}_{\geq 0}}} x_{1}^{i_{1}} \cdots x_{m}^{i_{m}}
$$

be the jth complete symmetric polynomial. Then

$$
G_{l}^{m}\left(q_{1}, \ldots, q_{m}\right)=\sum_{j=0}^{l} h_{j}\left(q_{1}, q_{1} q_{2}, \ldots, q_{1} q_{2} \cdots q_{m}\right) .
$$

In particular,

$$
G_{\infty}^{m}\left(q_{1}, \ldots, q_{m}\right):=\lim _{l \rightarrow \infty} G_{l}^{m}\left(q_{1}, \ldots, q_{m}\right)=\prod_{k=1}^{m}\left(1-q_{1} q_{2} \cdots q_{k}\right)^{-1} .
$$

Proof. Since

$$
\begin{aligned}
h_{j}\left(q_{1}, q_{1} q_{2}, \ldots, q_{1} q_{2} \cdots q_{m}\right) & =\sum_{i_{1}+i_{2}+\cdots+i_{m}=j} q_{1}^{i_{1}+i_{2}+\cdots+i_{m}} q_{2}^{i_{2}+\cdots+i_{m}} \cdots q_{m}^{i_{m}} \\
& =\sum_{j=\lambda_{1} \geq \lambda_{2} \geq \cdots \geq \lambda_{m} \geq 0} q_{1}^{\lambda_{1}} q_{2}^{\lambda_{2}} \cdots q_{m}^{\lambda_{m}},
\end{aligned}
$$

(3.3) is clear from the definition (3.2). The second formula follows from (3.3) 
together with the fact that

$$
\sum_{j=0}^{\infty} h_{j}\left(x_{1}, \ldots, x_{m}\right) z^{j}=\prod_{k=1}^{m}\left(1-x_{k} z\right)^{-1} \quad(\text { see }[\mathrm{M}]) .
$$

As a corollary, we obtain the Euler product of $Z_{N}^{m}\left(t_{1}, \ldots, t_{m}\right)$.

Corollary 3.3. Let $m$ and $N$ be positive integers. Then

$$
Z_{N}^{m}\left(t_{1}, \ldots, t_{m}\right)=\prod_{p: \text { prime }}\left(\sum_{j=0}^{\operatorname{ord}_{p} N} h_{j}\left(p^{-t_{1}}, p^{-t_{1}-t_{2}}, \ldots, p^{-t_{1}-t_{2}-\cdots-t_{m}}\right)\right) .
$$

Further, for $\operatorname{Re} t_{j}>1(1 \leq j \leq m)$,

$$
Z_{\infty}^{m}\left(t_{1}, \ldots, t_{m}\right):=\sum_{n_{m}|\cdots| n_{1}} n_{1}^{-t_{1}} \cdots n_{m}^{-t_{m}}=\prod_{k=1}^{m} \zeta\left(t_{1}+\cdots+t_{k}\right) .
$$

Since

$$
\begin{aligned}
G_{l}^{\gamma}\left(q_{1}, \ldots, q_{m}\right) & =\sum_{\substack { 0 \leq n \leq l / \gamma_{1} \\
\begin{subarray}{c}{\gamma_{1} n \geq \lambda_{2} \geq \cdots \geq \lambda_{m} \geq 0 \\
\gamma_{j} \mid \lambda_{j}(2 \leq j \leq m){ 0 \leq n \leq l / \gamma _ { 1 } \\
\begin{subarray} { c } { \gamma _ { 1 } n \geq \lambda _ { 2 } \geq \cdots \geq \lambda _ { m } \geq 0 \\
\gamma _ { j } | \lambda _ { j } ( 2 \leq j \leq m ) } }\end{subarray}} q_{2}^{\gamma_{1} n} \cdots q_{m}^{\lambda_{m}} \\
& =\sum_{n=0}^{\left\lfloor l / \gamma_{1}\right\rfloor} q_{1}^{\gamma_{1} n} G_{\gamma_{1} n}^{\left(\gamma_{2}, \ldots, \gamma_{m}\right)}\left(q_{2}, \ldots, q_{m}\right)
\end{aligned}
$$

the recurrence equation among $G_{l}^{\gamma}\left(q_{1}, \ldots, q_{m}\right)$ 's can be obtained as follows.

LEMMA 3.4. For $\gamma=\left(\gamma_{1}, \ldots, \gamma_{m}\right) \in \mathbb{Z}_{>0}^{m}$, we have

$$
G_{l}^{\gamma}\left(q_{1}, \ldots, q_{m}\right)=\sum_{n=0}^{\left\lfloor l / \gamma_{1}\right\rfloor} q_{1}^{\gamma_{1} n} G_{\gamma_{1} n}^{\left(\gamma_{2}, \ldots, \gamma_{m}\right)}\left(q_{2}, \ldots, q_{m}\right)
$$

Here $\lfloor x\rfloor$ is the largest integer not exceeding $x$.

The following lemma shows that to study $G_{l}^{\gamma}\left(q_{1}, \ldots, q_{m}\right)$ it is enough to study the case where $\gamma_{1}, \ldots, \gamma_{m}$ are relatively prime. The proof is straightforward.

LEMMA 3.5. For $\gamma=\left(d c_{1}, \ldots, d c_{m}\right)$, we have

$$
G_{l}^{\gamma}\left(q_{1}, \ldots, q_{m}\right)=G_{\lfloor l / d\rfloor}^{\left(c_{1}, \ldots, c_{m}\right)}\left(q_{1}^{d}, \ldots, q_{m}^{d}\right)
$$

Let us calculate several $G^{\gamma}\left(q_{1}, \ldots, q_{m}\right)$ with special parameters $\gamma$.

ExAmple 3.1. Let $\gamma=(c, 1)$. Putting $d=\lfloor l / c\rfloor$, we see by Lemma 3.4 that

$$
\begin{aligned}
& G_{l}^{(c, 1)}\left(q_{1}, q_{2}\right) \\
& \quad=\frac{\left(1-q_{1}^{c(d+1)}\right)\left(1-\left(q_{1} q_{2}\right)^{c}\right)-q_{2}\left(1-q_{1}^{c}\right)\left(1-\left(q_{1} q_{2}\right)^{c(d+1)}\right)}{\left(1-q_{2}\right)\left(1-q_{1}^{c}\right)\left(1-\left(q_{1} q_{2}\right)^{c}\right)}
\end{aligned}
$$


Therefore

$$
Z_{\infty}^{(c, 1)}\left(t_{1}, t_{2}\right)=\zeta\left(t_{2}\right) \zeta\left(c t_{1}\right) \zeta\left(c\left(t_{1}+t_{2}\right)\right) \prod_{p: \text { prime }}\left(1-p^{-t_{2}}+p^{-c t_{1}-t_{2}}-p^{-c t_{1}-c t_{2}}\right)
$$

for $\operatorname{Re} t_{j}>1(j=1,2)$. Thus, we may have various Euler products of the form $\prod_{p \text { : prime }} H\left(p^{-s}, p^{-t}\right)$, where $H(S, T) \in 1+S \cdot \mathbb{C}[S, T]+T \cdot \mathbb{C}[S, T]$ arises from $Z_{\infty}^{(c, 1)}\left(t_{1}, t_{2}\right)$. Then

EXAmple 3.2. We calculate $G_{\infty}^{(c d, c, 1)}(q, q, q)$. Set $q_{1}=q_{2}=q$ in (3.4).

$$
G_{l}^{(c, 1)}(q, q)=\frac{\left(1-q^{c(d+1)}\right)\left(1-q+q^{c}-q^{c d+c+1}\right)}{(1-q)\left(1-q^{2 c}\right)} .
$$

It follows from Lemma 3.4 that

$$
G_{\infty}^{(c d, c, 1)}(q, q, q)=\frac{1}{(1-q)\left(1-q^{2 c}\right)}\left\{\frac{1-q+q^{c}}{1-q^{c d}}-\frac{q^{c}\left(1+q^{c}\right)}{1-q^{2 c d}}+\frac{q^{2 c+1}}{1-q^{3 c d}}\right\} .
$$

In particular, putting $d=1$, we obtain

$$
\begin{aligned}
Z_{\infty}^{(c, c, 1)}(s) & =\sum_{n_{3}\left|n_{2}^{c}\right| n_{1}^{c}}\left(n_{1}^{c} n_{2}^{c} n_{3}\right)^{-s} \\
& =\zeta(s) \zeta(2 c s) \zeta(3 c s) \prod_{p: \text { prime }}\left(1-p^{-s}+p^{-c s}-p^{-(c+1) s}+p^{-2 c s}\right)
\end{aligned}
$$

4. Multiple zeta functions and powerful numbers. In this section, we study $Z_{\infty}^{\gamma}(s)$ for $\gamma=(k, k, \ldots, k, 1)$ in connection with a certain generalized notion of powerful numbers.

Let us first recall the definition of powerful numbers. A positive number $n$ is called $k$-powerful if $\operatorname{ord}_{p} n \geq k$ for any prime number $p$ unless $\operatorname{ord}_{p} n=0$ (see, e.g., [IS], [I]). Extending this, we arrive at a new notion: a positive integer $n$ is said to be an l-step $k$-powerful number if either $\operatorname{ord}_{p} n=0, k, 2 k, \ldots,(l-1) k$ or $\operatorname{ord}_{p} n \geq l k$ for any prime number $p$. Clearly, if $n$ is an $l$-step $k$-powerful number, then $n$ is a $j$-step $k$-powerful number for each $j(1 \leq j \leq l)$. In particular, 1-step $k$-powerful numbers are nothing but the usual $k$-powerful numbers. Note also that every natural number is an $l$-step 1-powerful number for any $l$; this agrees with the claim for $k=1$ in Theorem 4.8 below.

As an example of $l$-step $k$-powerful numbers, we list the first few 2 -step 2-powerful numbers: $1,4,9,16,25,32,36,49,64,81,100,121,128,144$, $169,196,225,243, \ldots$ Note that in general an $l$-step $k$-powerful number $n$ has the canonical representation: $n=a_{1}^{k} a_{2}^{2 k} \cdots a_{l}^{(l-1) k} m$, where $a_{1}, \ldots, a_{l}$ are square-free, $m$ is $(l k)$-powerful and $\operatorname{gcd}\left(a_{1}, \ldots, a_{l}, m\right)=1$. Note also that a $k$-powerful number $m$ is uniquely expressed as $m=b_{1}^{k} b_{2}^{k+1} \cdots b_{k}^{2 k-1}$ if we 
stipulate that $b_{2}, \ldots, b_{k}$ are all square-free. Put

$$
f_{k, l}(n):= \begin{cases}1 & \text { if } n \text { is an } l \text {-step } k \text {-powerful number } \\ 0 & \text { otherwise }\end{cases}
$$

for a positive integer $n$. Define also $F_{k, l}(s):=\sum_{n=1}^{\infty} f_{k, l}(n) n^{-s}$. The arithmetic function $f_{k, l}(n)$ is multiplicative with respect to $n$. Note that $F_{1, l}(s)=$ $\zeta(s)$ for any $l$. We show that $Z_{\infty}^{\gamma}(s)$ is represented by the product of the Riemann zeta functions times $F_{k, l}(s)$.

TheOrEm 4.1. Let $k, l$ be positive integers, and put $\gamma=(\overbrace{k, k, \ldots, k}^{l}, 1)$. Then

$$
\begin{aligned}
Z_{\infty}^{\gamma}(s) & =\sum_{n_{l+1}\left|n_{l}^{k}\right| \cdots \mid n_{1}^{k}}\left(n_{1}^{k} \cdots n_{l}^{k} n_{l+1}\right)^{-s} \\
& =F_{k, l}(s) \prod_{j=2}^{l+1} \zeta(j k s) \quad(\operatorname{Re} s>1) .
\end{aligned}
$$

In particular, $Z_{\infty}^{m}(s)=\prod_{j=1}^{m} \zeta(j s)$.

To prove the theorem, we need the following two lemmas.

Lemma 4.2. Let $\gamma=(\overbrace{k, k, \ldots, k}^{l} 1)$. Then

$$
G_{\infty}^{\gamma}(q)=G_{\infty}^{\gamma}(\overbrace{q, q, \ldots, q}^{l+1})=\prod_{j=1}^{l+1} \frac{1}{1-q^{j k}} \cdot \frac{1-q+q^{l k+1}-q^{k(l+1)}}{1-q} .
$$

Proof. By definition, we have

$$
\begin{aligned}
& G_{\infty}^{(k, \ldots, k, 1)}(q)=\sum_{\substack{\lambda_{1} \geq \ldots \geq \lambda_{l} \geq \lambda_{l+1} \geq 0 \\
k \mid \lambda_{j}(1 \leq j \leq l)}} q^{\lambda_{1}+\cdots+\lambda_{l}+\lambda_{l+1}}=\sum_{n=0}^{\infty} q^{n} \sum_{\substack{\lambda_{1} \geq \cdots \geq \lambda_{l} \geq n \\
k \mid \lambda_{j}(1 \leq j \leq l)}} q^{\lambda_{1}+\cdots+\lambda_{l}} \\
& =\sum_{\substack{\lambda_{1} \geq \cdots \geq \lambda_{l} \geq 0 \\
k \mid \lambda_{j}(1 \leq j \leq l)}} q^{\lambda_{1}+\cdots+\lambda_{l}}+\sum_{a=0}^{\infty} \sum_{b=1}^{k} q^{a k+b} \sum_{\substack{\lambda_{1} \geq \cdots \geq \lambda_{l} \geq a k+b \\
k \mid \lambda_{j}(1 \leq j \leq l)}} q^{\lambda_{1}+\cdots+\lambda_{l}} \\
& =\sum_{\mu_{1} \geq \cdots \geq \mu_{l} \geq 0} q^{k\left(\mu_{1}+\cdots+\mu_{l}\right)}+\sum_{a=0}^{\infty} \sum_{b=1}^{k} q^{a k+b} \sum_{\mu_{1} \geq \cdots \geq \mu_{l} \geq a+1} q^{k\left(\mu_{1}+\cdots+\mu_{l}\right)} \\
& =G_{\infty}^{l}\left(q^{k}\right)+\sum_{a=0}^{\infty} q^{a k} \sum_{b=1}^{k} q^{b} \sum_{\nu_{1} \geq \cdots \geq \nu_{l} \geq 0} q^{k\left\{\left(\nu_{1}+a+1\right)+\cdots+\left(\nu_{l}+a+1\right)\right\}} \\
& =G_{\infty}^{l}\left(q^{k}\right)+q^{l k}\left(\sum_{a=0}^{\infty} q^{k(l+1) a}\right)\left(\sum_{b=1}^{k} q^{b}\right)\left(\sum_{\nu_{1} \geq \cdots \geq \nu_{l} \geq 0} q^{k\left(\nu_{1}+\cdots+\nu_{l}\right)}\right) .
\end{aligned}
$$


Since $G_{\infty}^{l}(q)=\prod_{j=1}^{l}\left(1-q^{j}\right)^{-1}$, we have

$$
\begin{aligned}
G_{\infty}^{(k, \ldots, k, 1)}(q) & =\prod_{j=1}^{l} \frac{1}{1-q^{j k}}\left(1+\frac{q^{k l}}{1-q^{k(l+1)}} \cdot \frac{q\left(1-q^{k}\right)}{1-q}\right) \\
& =\prod_{j=1}^{l+1} \frac{1}{1-q^{j k}} \cdot \frac{1-q+q^{l k+1}-q^{k(l+1)}}{1-q} .
\end{aligned}
$$

This proves the assertion.

The following lemma is easily obtained.

Lemma 4.3. We have

$$
\frac{1-q+q^{l k+1}-q^{k(l+1)}}{1-q}=\left(1-q^{k}\right)\left(1+q^{k}+q^{2 k}+\cdots+q^{(l-1) k}+q^{l k} \sum_{j=0}^{\infty} q^{j}\right) .
$$

Proof of Theorem 4.1. It follows from Lemmas 4.2 and 4.3 that

$$
\begin{aligned}
Z_{\infty}^{(k, \ldots, k, 1)}(s)=\prod_{p: \text { prime }} G_{\infty}^{(k, \ldots, k, 1)}\left(p^{-s}\right) \\
=\prod_{p: \text { prime }}\left(\prod_{j=2}^{\infty} \frac{1}{1-p^{-j k s}}\right) \\
\quad \times \prod_{p: \text { prime }}\left(1+p^{-k s}+p^{-2 k s}+\cdots+p^{-(l-1) k s}+p^{-l k s}+p^{-(l k+1) s}+\cdots\right) \\
=\prod_{j=2}^{l+1} \zeta(j k s) \cdot F_{k, l}(s) . \mathbf{-}
\end{aligned}
$$

Now we determine when the Dirichlet series $F_{k, l}(s)$ can be meromorphically extended to $\mathbb{C}$. We recall the following result of Estermann's $[\mathrm{E}]$ (see $[\mathrm{K}]$ for a generalization). A polynomial $f(T) \in 1+T \cdot \mathbb{C}[T]$ is said to be unitary if there is a unitary matrix $M$ such that $f(T)=\operatorname{det}(1-M T)$. Then:

Lemma 4.4. For $f(T) \in 1+T \cdot \mathbb{C}[T]$, put $L(s, f)=\prod_{p: \text { prime }} f\left(p^{-s}\right)$.

(i) $f(T)$ is unitary if and only if $L(s, f)$ can be extended as a meromorphic function on $\mathbb{C}$.

(ii) $f(T)$ is not unitary if and only if $L(s, f)$ can be extended as a meromorphic function in $\operatorname{Re} s>0$ with the natural boundary $\operatorname{Re} s$ $=0$; each point on $\operatorname{Re} s=0$ is a limit point of poles of $L(s, f)$ in $\operatorname{Re} s>0$. 
Since

$$
\begin{aligned}
F_{k, l}(s) & =\prod_{p: \text { prime }}\left(1+p^{-k s}+p^{-2 k s}+\cdots+p^{-(l-1) k s}+p^{-l k s} \sum_{j=0}^{\infty} p^{-j s}\right) \\
& =\zeta(s) \zeta(k s) \prod_{p: \text { prime }}\left(1-p^{-s}+p^{-(l k+1) s}-p^{-k(l+1) s}\right)
\end{aligned}
$$

by Lemma 4.3, we only have to see whether the polynomial $G_{k, l}(T):=1-T+$ $T^{l k+1}-T^{k(l+1)}$ is unitary or not. The polynomial $G_{k, l}(T)$ can be expressed as $G_{k, l}(T)=\left(1-T^{k}\right) H_{k, l}(T)$ with $H_{k, l}(T):=1+\left(T^{k}-T\right) \sum_{j=0}^{l-1} T^{k j}$.

Proposition 4.5. The polynomial $G_{k, l}(T)$ is unitary if and only if $k=$ 1,2 .

In order to prove this proposition, we need the following two lemmas.

LEMMA 4.6. Let $k \geq 3$. Then the unitary root $\alpha$ (i.e. $|\alpha|=1$ ) of the polynomial $G_{k, l}(T)$ must satisfy $\alpha^{k}=1$ or $\alpha^{k-2}=1$.

Proof. Let $\alpha=e^{2 \pi i \theta} \neq 1(\theta \in \mathbb{R})$ be a unitary root of $G_{k, l}(T)$. Since

$$
G_{k, l}(T) /(1-T)=1+T^{l k+1}\left(1-T^{k-1}\right) /(1-T),
$$

we have $1+\alpha^{l k+1}\left(1-\alpha^{k-1}\right) /(1-\alpha)=0$ so that

$$
\left|\left(1-\alpha^{k-1}\right) /(1-\alpha)\right|=\left|\alpha^{-(l k+1)}\right|=1 .
$$

Hence $\operatorname{Re} \alpha^{k-1}=\operatorname{Re} \alpha$, that is,

$$
\cos 2 \pi(k-1) \theta-\cos 2 \pi \theta=-2 \sin \pi k \theta \sin \pi(k-2) \theta=0 .
$$

Thus we conclude that either $k \theta \in \mathbb{Z}$ or $(k-2) \theta \in \mathbb{Z}$. This proves the lemma.

Lemma 4.7. Let $k \geq 3$. Suppose that a complex number $\alpha$ satisfies $\alpha^{k-2}$ $=1$. Then $G_{k, l}^{\prime \prime}(\alpha) \neq 0$.

Proof. Since

$$
G_{k, l}^{\prime \prime}(T)=(l k+1) l k T^{l(k-2)+2 l-1}-(k l+k)(k l+k-1) T^{(k-2)(l+1)+2 l},
$$

if we assume that $\alpha$ satisfies $G_{k, l}^{\prime \prime}(\alpha)=0$ and $\alpha^{k-2}=1$, we have

$$
G_{k, l}^{\prime \prime}(\alpha)=(l k+1) l k \alpha^{2 l-1}-(k l+k)(k l+k-1) \alpha^{2 l}=0 .
$$

This shows that $\alpha=l(l k+1) /(l+1)(k l+k-1)$, which contradicts $\alpha^{k-2}=1$. Hence the assertion follows.

Proof of Proposition 4.5. Let $l$ be a positive integer. Since the unitarity of $G_{k, l}(T)$ and of $H_{k, l}(T)$ are equivalent, it suffices to check the latter. It is clear that $H_{k, l}(T)$ is a unitary polynomial when $k=1,2$. Actually we have $H_{1, l}(T)=1$ and $H_{2, l}(T)=1-T+T^{2 l+1}-T^{2 l+2}=(1-T)\left(1+T^{2 l+1}\right)$, which are indeed unitary. 
Suppose $k \geq 3$. If $G_{k, l}(T)$ is unitary, then every root of $H_{k, l}(T)$ satisfies $\alpha^{k}=1$ or $\alpha^{k-2}=1$ by Lemma 4.6. However, if $\alpha^{k}=1$ we immediately see that $H_{k, l}(\alpha)=1+(1-\alpha) l$. Thus, $H_{k, l}(\alpha)$ cannot be 0 because of the unitarity of $\alpha$. Thus any root of $H_{k, l}(T)$ must satisfy $\alpha^{k-2}=1$ and $\alpha^{k} \neq 1$. By Lemma 4.7, the multiplicity of these roots of $H_{k, l}(T)$ is at most 2. Since $H_{k, l}(T)$ is assumed to be unitary and $H_{k, l}(1) \neq 0$, it follows that $2(k-3) \geq \operatorname{deg} H_{k, l}(T)=l k$. This is possible only when $l=1$. Therefore it is enough to prove that $H_{k, 1}(T)$ is not unitary for $k \geq 3$. We put $H_{k}(T)=$ $H_{k, 1}(T)=1-T+T^{k}$ for simplicity. If $k$ is odd, then $H_{k}(T)$ has a real root in the interval $(-2,-1)$ since $H_{k}(-1)=1>0$ and $H_{k}(-2)=3-2^{k}<0$. This implies $H_{k}(T)$ is not unitary.

Thus, it remains to consider the case where $k$ is even and $k \geq 4$. Suppose that $H_{k}(T)$ is unitary and let $e^{i \theta}(-\pi<\theta \leq \pi)$ be its unitary root. Then $\cos k \theta=\cos \theta-1$ and $\sin k \theta=\sin \theta$. Since $1=\sin ^{2} k \theta+\cos ^{2} k \theta=2-2 \cos \theta$, we have $\theta= \pm \pi / 3$. Further, since $1=\cos ^{2} \theta+\sin ^{2} \theta=(\cos k \theta+1)^{2}+$ $\sin ^{2} k \theta=2 \cos k \theta+2$, we have $\cos (k \pi / 3)=-1 / 2$. Hence either $k \equiv 2$ or 4 $(\bmod 6)$. On the other hand, since $1=(\cos \theta-\cos k \theta)^{2}+(\sin \theta-\sin k \theta)^{2}=$ $2-2 \cos (k-1) \theta$, we have $\cos ((k-1) \pi / 3)=1 / 2$. It follows that either $k \equiv 0$ or $2(\bmod 6)$. Thus $k \equiv 2(\bmod 6)$.

Now we show that every unitary root of $H_{k}(T)$ is simple. If we assume that $\beta$ is a multiple root of $H_{k}(T)$, it follows that $\beta^{k}-\beta+1=0$ and $k \beta^{k-1}-1=0$. Then $|\beta|=k^{-1 /(k-1)}$ and $\beta=k \beta^{k}$ by the second equation. On the other hand, by the first equation, $1=\beta-\beta^{k}=(k-1) \beta^{k}$ so that $|\beta|=(k-1)^{-1 / k}$. Therefore $k^{k}=|\beta|^{-k(k-1)}=(k-1)^{k-1}$, which contradicts the unitarity of $H_{k}(T)$. This completes the proof of the proposition.

Finally, we obtain the following generalization of the result in [IS] concerning powerful numbers. The proof follows immediately from Lemma 4.4 and Proposition 4.5.

TheOREM 4.8. Let $k$ and $l$ be positive integers. When $k=1,2$ we have

$$
F_{1, l}(s)=\zeta(s), \quad F_{2, l}(s)=\frac{\zeta(2 s) \zeta((2 l+1) s)}{\zeta(2(2 l+1) s)} .
$$

When $k \geq 3, F_{k, l}(s)$ is meromorphic in $\operatorname{Re} s>0$ and has a natural boundary $\operatorname{Re} s=0$.

Corollary 4.9. Let $Z_{\infty}^{(k, \ldots, k, 1)}(s)$ be as in Theorem 4.1. Then for $k=$ 1,2 we have

$$
Z_{\infty}^{(1, \ldots, 1,1)}(s)=\prod_{j=1}^{l+1} \zeta(j s), \quad Z_{\infty}^{(2, \ldots, 2,1)}(s)=\frac{\zeta((2 l+1) s)}{\zeta(2(2 l+1) s)} \prod_{j=1}^{l+1} \zeta(2 j s) .
$$

For $k \geq 3, Z_{\infty}^{(k, \ldots, k, 1)}(s)$ can be meromorphically extended to $\operatorname{Re} s>0$ with a natural boundary $\operatorname{Re} s=0$. 
5. Closing remarks. We give two remarks.

- The isomorphism classes of abelian groups $A$ of order $n$ are indexed by the map $\boldsymbol{\lambda}$ from the set of all prime numbers to that of partitions such that

$$
n=\prod_{p: \text { prime }} p^{|\lambda(p)|}, \quad A \cong \bigoplus_{p: \text { prime }} \bigoplus_{j=1}^{\ell(\lambda(p))} \mathbb{Z} / p^{\lambda_{j}(p)} \mathbb{Z},
$$

where $|\boldsymbol{\lambda}(p)|$ and $\ell(\boldsymbol{\lambda}(p))$ are the size and length of the partition $\boldsymbol{\lambda}(p)=$ $\left(\boldsymbol{\lambda}_{j}(p)\right)_{j \geq 1}$ respectively. The multiple finite Riemann zeta function is expressed also as $Z_{N}^{m}(s)=\sum_{n \mid N^{m}} g_{N}^{m}(n) n^{-s}$. Here $g_{N}^{m}(n)$ is the number of the isomorphism classes of abelian groups of order $n$, parametrized by $\boldsymbol{\lambda}$ such that $\boldsymbol{\lambda}_{1}(p) \leq m$ and $\ell(\boldsymbol{\lambda}(p)) \leq \operatorname{ord}_{p} N$ for all $p$. It is clear that $g_{N}^{m}(n)$ is multiplicative with respect to $n$ and $N$. Put $g_{\infty}^{m}(n):=\lim _{N \rightarrow \infty} g_{N}^{m}(n)$. Then $g_{\infty}^{m}(n)$ is the number of isomorphism classes of abelian groups $A$ of order $n$ which are direct sums of $p$-groups $A_{p}$ such that $p^{m} A_{p}=0$ for $p \mid n$.

We now study the asymptotic average for $g_{\infty}^{m}(n)$ and $Z_{n}^{m}(\sigma)(\sigma \in \mathbb{R})$ with respect to $n$. Thus we need the Tauberian theorem below (see, e.g., [MM]).

Lemma 5.1. Let $F(t)=\sum_{n=1}^{\infty} a_{n} n^{-t}$ be a Dirichlet series with nonnegative real coefficients which converges absolutely for $\operatorname{Re} t>\beta$. Suppose that $F(t)$ has a meromorphic continuation to the region $\operatorname{Re} t \geq \beta$ with a pole of order $\alpha+1$ at $t=\beta$ for some $\alpha \geq 0$. Put

$$
c:=\frac{1}{\alpha !} \lim _{t \rightarrow \beta}(t-\beta)^{\alpha+1} F(t) .
$$

Then

$$
\sum_{n \leq x} a_{n}=(c+o(1)) x^{\beta}(\log x)^{\alpha} \quad \text { as } x \rightarrow \infty .
$$

Using this lemma, we easily obtain the following results. Let $m$ be a positive integer.

1. We have $\sum_{n \leq x} g_{\infty}^{m}(n)=(\zeta(2) \zeta(3) \cdots \zeta(m)+o(1)) x$ as $x \rightarrow \infty$. In other words, the asymptotic average of $g_{\infty}^{m}(n)$ with respect to $n$ is the product $\zeta(2) \zeta(3) \cdots \zeta(m)$.

2. For a fixed $\sigma>0$, when $x \rightarrow \infty$,

$$
\begin{aligned}
\sum_{n \leq x} Z_{n}^{m}(\sigma) & =(\zeta(\sigma+1) \zeta(2 \sigma+1) \cdots \zeta(m \sigma+1)+o(1)) x, \\
\sum_{n \leq x} Z_{n}^{m}(-\sigma) & =(\zeta(\sigma+1) \zeta(2 \sigma+1) \cdots \zeta(m \sigma+1)+o(1)) x^{1+m \sigma}, \\
\sum_{n \leq x} Z_{n}^{m}(0) & =\sum_{n \leq x} \prod_{p: \text { prime }}\left(\begin{array}{c}
\operatorname{ord}_{p} n+m \\
m
\end{array}\right)=\left(\frac{1}{m !}+o(1)\right) x(\log x)^{m} .
\end{aligned}
$$


Actually, since $\zeta(s)$ has a single pole at $s=1$ and $\operatorname{Res}_{s=1} \zeta(s)=1$, the first assertion follows from (2.6) and Lemma 5.1. Next, fix $\sigma \in \mathbb{R}$. By (2.6), we have

$$
\zeta^{m}(\sigma, t)=\sum_{n=1}^{\infty} Z_{n}^{m}(\sigma) n^{-t}=\prod_{k=0}^{m} \zeta(t+k \sigma) .
$$

This shows that the abscissa of absolute convergence of the Dirichlet series $\zeta^{m}(\sigma, t)$ is $t=\max \{1,1-m \sigma\}$. Hence the remaining formulas follow similarly.

REMARK 5.1. For $g(n):=\lim _{m \rightarrow \infty} g_{\infty}^{m}(n)$, it is well known (see [A]) that

$$
\sum_{n \leq x} g(n)=\left(\prod_{k=2}^{\infty} \zeta(k)\right) x+O(\sqrt{x}) .
$$

Since $Z_{n}^{1}(0)=d(n):=\sum_{d \mid n} 1$, we have $\sum_{n \leq x} d(n) \sim x \log x$. It is also well known (see [Z]) that there exists a constant $C$ such that $\sum_{n \leq x} d(n)=$ $x \log x+C x+O(\sqrt{x})$ in an elementary way.

- We define a multiple Eisenstein series with parameter $s$ of type $m$ by

$$
E_{s}^{m}(q)=\sum_{n=1}^{\infty} Z_{n}^{m}(1-s) q^{n} .
$$

We sometimes write $E_{s}^{m}(\tau)$ instead of $E_{s}^{m}(q)$ when $q=e^{2 \pi i \tau}$ with $\tau \in \mathbb{C}$, $\operatorname{Im} \tau>0$. It is obvious that $E_{k}^{1}(q)$ is (essentially) the usual holomorphic Eisenstein series of weight $k$. In this remark we make an experimental study of $E_{s}^{m}(q)$ when $m=2$. First, the following is easily obtained:

Lemma 5.2. We have $E_{s+1}^{2}(q)=\sum_{l=1}^{\infty} \sum_{N=1}^{\infty} \sigma_{s}(N) N^{s} q^{N l}$.

Recall now the Fourier expansion of $E_{k+1}(\tau)$ of weight $k+1$ with $k$ being odd:

$$
\begin{aligned}
E_{k+1}(\tau) & =1+\frac{1}{\zeta(k+1)} \cdot \frac{(2 \pi i)^{k+1}}{k !} \sum_{n=1}^{\infty} \sigma_{k}(n) q^{n} \\
( & \left.=1+\frac{1}{\zeta(k+1)} \cdot \frac{(2 \pi i)^{k+1}}{k !} E_{k+1}^{1}(\tau)\right) .
\end{aligned}
$$

Take the $k$ th derivative of $E_{k+1}(\tau)$. Then, if $k$ is odd, by Lemma 5.2 we immediately get

$$
E_{k+1}^{2}(\tau)=\frac{\zeta(k+1) k !}{(2 \pi i)^{2 k+1}} \sum_{l=1}^{\infty}\left(\frac{d^{k}}{d \tau^{k}} E_{k+1}\right)(l \tau) .
$$


There is also an expression of $E_{k+1}^{2}(\tau)$ similar to $E_{k+1}(\tau)$, when $k$ is odd:

$$
E_{k+1}^{2}(\tau)=-\frac{(2 k) !}{(2 \pi i)^{2 k+1}} \sum_{l=1}^{\infty} \sum_{\substack{(c, d)=1 \\ c>0}} \sigma_{k}(c l) l^{-2 k-1}(c \tau+d)^{-2 k-1} \text {. }
$$

\section{References}

[A] G. E. Andrews, Number Theory, Dover, 1994.

[AAR] G. E. Andrews, R. Askey and R. Roy, Special Functions, Encyclopedia Math. Appl. 71, Cambridge Univ. Press, Cambridge, 1999.

[BCKV] D. Bump, K.-K. Choi, P. Kurlberg and J. Vaaler, A local Riemann hypothesis, Math. Z. 233 (2000), 1-19.

[E] T. Estermann, On certain functions represented by Dirichlet series, Proc. London Math. Soc. 27 (1928), 435-448.

[I] A. Ivić, The Riemann Zeta-Functions. The Theory of the Riemann Zeta-Function with Applications, Wiley, 1985.

[IS] A. Ivić and P. Shiu, The distribution of powerful integers, Illinois J. Math. 26 (1982), 576-590.

[K] N. Kurokawa, On the meromorphy of Euler products $(I),(I I)$, Proc. London Math. Soc. 53 (1986), 1-47, 209-236.

[KMW] N. Kurokawa, S. Matsuda and M. Wakayama, Gamma factors and functional equations of higher Riemann zeta functions, preprint (2003).

[KW] N. Kurokawa and M. Wakayama, Higher Selberg zeta functions, Comm. Math. Phys. 247 (2004), 447-466.

[M] I. G. Macdonald, Symmetric Functions and Hall Polynomials, 2nd ed., Oxford Univ. Press, 1995.

[MM] M. R. Murty and V. K. Murty, Non-vanishing of L-functions and Applications, Progr. Math. 157, Birkhäuser, 1997.

[Z] D. Zagier, Zetafunktionen und quadratische Körper, Springer, Berlin, 1981.

Department of Mathematical Science

University of the Ryukyus

Nishihara, Okinawa

903-0213 Japan

E-mail: kimoto@math.u-ryukyu.ac.jp

Department of Mathematics

Tokyo Institute of Technology

Oh-okayama Meguro-ku, Tokyo

152-0033 Japan

E-mail: kurokawa@math.titech.ac.jp
Graduate School of Mathematics Kyushu University Hakozaki Higashi-ku, Fukuoka 812-8581 Japan E-mail: ma203029@math.kyushu-u.ac.jp

Faculty of Mathematics Kyushu University Hakozaki Higashi-ku, Fukuoka 812-8581 Japan E-mail: wakayama@math.kyushu-u.ac.jp

Received on 13.4.2004

and in revised form on 18.5.2004 